\title{
Vibratory drilling with digital adaptive control
}

\author{
Ilya Ivanov ${ }^{1, *}$, Ivan Pleshcheev ${ }^{1}$, and Andrey Larkin ${ }^{1}$ \\ ${ }^{1}$ Bauman Moscow State Technical University, Department of Applied Mechanics, 105005 2-Ya \\ Baumanskaya ul., 5, Moscow, Russia
}

\begin{abstract}
High standards and efficiency of deep hole drilling used for hard-to-machine metals and alloys could not be achieved if chips are not segmented in the cutting zone. Chip control could be achieved through transmission of harmonic vibrations to the drill in direction of its rotating axis. One way to maintain these vibrations is to replace a drill chuck with a special self-vibratory drilling head, which includes an elastic element that allows axial movement of the tool. The right stiffness value of an elastic element and appropriate machining conditions could lead to oscillation self-excitation of a drill due to the regenerative mechanism. It is advisable to support this mechanism with a control action defined within the feedback loop, which provides process quality necessary for chip control in the broad range of process parameters. This work analyzes adaptive control algorithm for vibratory drilling process dynamics where control action over an oscillating system is proportional to drill axial vibrational velocity, and a feedback gain is determined in the adaptation loop. Dynamics modeling of a closed-loop nonlinear system "elastic system-machining process-control system" is carried out for cases with or without control proving effectiveness of control algorithm. The model was used to analyze how control system analog-to-digital conversion parameters influence vibratory process quality. Requirements, which ensure control system achieves a control aim, were approximately stated for capacity and response limit for analog-to-digital conversion.
\end{abstract}

\section{Introduction}

During deep hole drilling of hard-to-machine metals, flow chips may wrap the tool. This could cause temperature increase in the cutting zone, drill jamming and failure, or damage to the treated bore surface. It is reasonable to crush chips during treatment. This way drilling could go on uninterrupted improving productivity and quality of treatment.

One way of chip control is to transmit vibrations to the drill endlong. If the cutting edge of a drill is regularly ejected from the cutting zone, the chips could be segmented and easily removable from the bore. In study [1], the authors stated the required amplitude and frequency of oscillations to crush the chips. To ensure chip control, minimum peak-to-peak value should be equal to the tool feed per cutting edge.

Along with a method of strict motion specification for a tool, there is a technique based on recovery of primary cutting motion energy to sustain axial vibrations of a drill. In this

* Corresponding author: ivanovilig@gmail.com 
case, a drill is fixed into a special self-vibratory drilling head [2] (Fig. 1), which includes an elastic element ensuring mechanical compliance of a system in axial direction. Drill and moving (in axial direction) member of the self-vibratory drilling head can shift relative to a fixed part of the head and spindle. When certain requirements for stiffness of an elastic element and treatment modes are met, there is a possibility of self-excitation of drill oscillations due to regenerative mechanism [3].

A review of studies, which examine various models and methods of analysis of selfexcited vibrations during drilling, is considered in [4]. Axial oscillations of a tool could cause dynamic instability of a non-deformed tool axis form, which could adversely influence service life of a drill and possibility to maintain a mode of axial self-vibrations. This issue is addressed in [5], where the authors give an example of dynamics simulation of vibratory drilling with regard to mechanical compliance of a tool as a generalized threedimensional model; however, no comparison with the experiment is made.

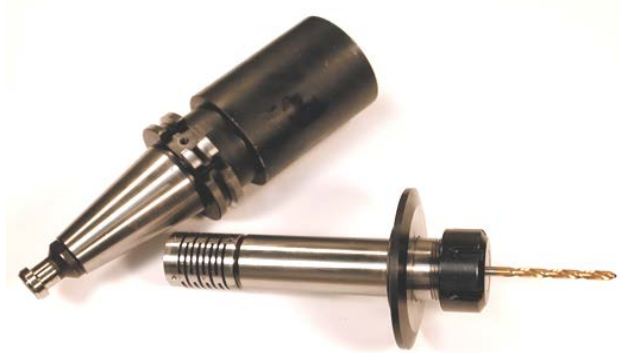

Fig. 1. A picture of a vibratory drilling head with a built-in elastic element for cutting [6]

The studies $[6,7]$ show that amplitude and frequency of self-vibrations are defined by dynamic properties of a self-vibratory drilling head, specified machining conditions, properties of the treated material and drill geometries. In restrained terms, properties of self-vibrations may be either insufficient or excessive to ensure crushing of chips. Dynamic characteristics of self-vibratory drilling head and machining conditions require fine adjustment. When there is no accurate data on damping of a system, possible changes to properties of a tool due to wear, or cutting conditions when drilling, reliability rate of selfexcited oscillations maintenance is low.

A possible corrective measure for self-vibratory drilling heads could be supporting selfoscillatory excitation mechanism by external control action [8] built-in the feedback loop. The method relies on energy supply from control system to oscillating system, if oscillations are weak for crushing chips; in a reverse situation, control system limits amplitude of oscillations. Adaptive control systems meet this requirement. Certain issues of operation stability of designed adaptive control systems for vibratory drilling process dynamics are identified in $[9,10]$. Basically, there is no sufficient research on control action support of axial vibrations of a tool.

This paper examines strategies of vibratory drilling control with adaptation of feedback ratio by peak-to-peak value. In part 2, derivation of model's equations is presented. In part 3 , a control strategy is described and influence of control introduction on vibratory process quality is shown. In part 4, results of dynamics simulation of a system with different values of analog-to-digital conversion parameters are introduced. In part 5, the conclusions are drawn. 


\section{Mathematic dynamics model of vibratory drilling process with control}

A computational scheme is given is Fig. 2, where $x(\mathrm{t})$ is an axial coordinate of a tool; $k, d$ are, respectively, stiffness and damping of tool fixture; $m$ is mass of a moving member of a self-vibratory drilling head and tool; $a$ is feed per cutting edge; $F_{c}$ is cutting thrust force; $x_{a}$ is actuator extension. Equations describing the dynamics of vibratory drilling process with control are established in [9] and are given in this paper in dimensionless form without derivation:

$$
\begin{gathered}
\frac{1}{(2 \pi p)^{2}} \ddot{q}+\frac{\zeta}{\pi p} \dot{q}+q=P_{c}+q_{0} \\
\eta(\tau)=[\Lambda(\tau-1)+1-q(\tau)] H[\Lambda(\tau-1)+1-q(\tau)] \\
P_{c}=k_{c} \eta^{r} \\
\Lambda(\tau)=\Lambda(\tau-1)+1-\eta(\tau)
\end{gathered}
$$

where $q$ is dimensionless axial coordinate of a tool, in fractions of feed per cutting edge; $p$ is natural frequency of self-vibratory drilling head $\sqrt{\mathrm{k} / \mathrm{m}}$ to tooth pass frequency ratio; $\zeta$ is dimensionless damping of a system; $P_{c}$ is dimensionless cutting force in fractions of product of elastic element stiffness by feed; $q_{0}$ is dimensionless kinematic excitation, in fractions of feed; $\eta$ is dimensionless thickness of cut layer, in fractions of feed; $\tau$ is dimensionless time, in fractions of tool turn onto cutting edge; $\Lambda$ is dimensionless axial coordinate of treated surface; $k_{c}, r$ are empirical constants defined by properties of treated material, diameter and geometry of the drill cutting edge. (1) is a dynamics model of selfvibratory drilling head and tool, (2), (4) are models of surface geometry generation, (3) is a phenomenological model of cutting forces.

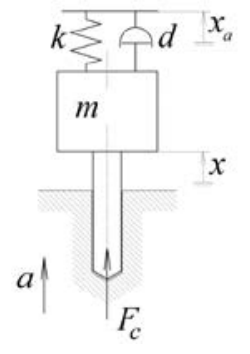

Fig. 2. Computational scheme of vibratory drilling process dynamics with control.

Obtained system of equations (1)-(4) is nonlinear with delayed argument included in the finite equations (2), (4); it is solved numerically. For further calculations assume $k_{c}=0.3, p$ $=1.5, \zeta=0.1$, found for drilling of steel 30 with a tool of $2 \mathrm{~mm}$ diameter and feed of 0.2 $\mathrm{mm} /$ cutting edge at $6000 \mathrm{rpm}$; natural frequency of self-vibratory drilling head is $300 \mathrm{~Hz}$, elastic element stiffness is $7100 \mathrm{~N} / \mathrm{mm}$.

Dynamic behavior of a tool while drilling is generally characterized by time dependence of drill movement and cutting force. We need to characterize oscillatory process in whole, to analyze the influence of process parameters (in this paper $-p, k_{c}$ ) and control algorithm 
parameters on oscillations of a tool. Peak-to-peak value of steady process is chosen as such characteristic.

\section{Control strategy}

Let us work out control action proportional to vibration velocity of a tool:

$$
q_{0}=k_{y} b \dot{q}
$$

where $k_{y}$ is a dimensionless gain ratio of control signal, $b$ is a feedback ratio defined by controller. While a digital controller is assumed to be used, $b$ may take a discrete sequence of values. For example, for 10-bit digital-to-analog converter it could be $(0 \ldots 1023)$ or $(-512 \ldots 511)$. Gain ratio is tried based on stability and quality of closed-loop system operation, «elastic system-machining process-control system», here and hereafter is assumed to be $k_{y}=5 \cdot 10^{-5}$.

Fig. 3 presents a system flowchart of "elastic system-machining process-control system". Proposed system is adaptive, i.e. feedback ratio $b$ could be modified to provide specified peak-to-peak value. If oscillations are not directly excited in the system, $b$ should take positive values ensuring energy supply to the system. If excessive excitation of oscillations takes place in the system, $b$ should take negative values.

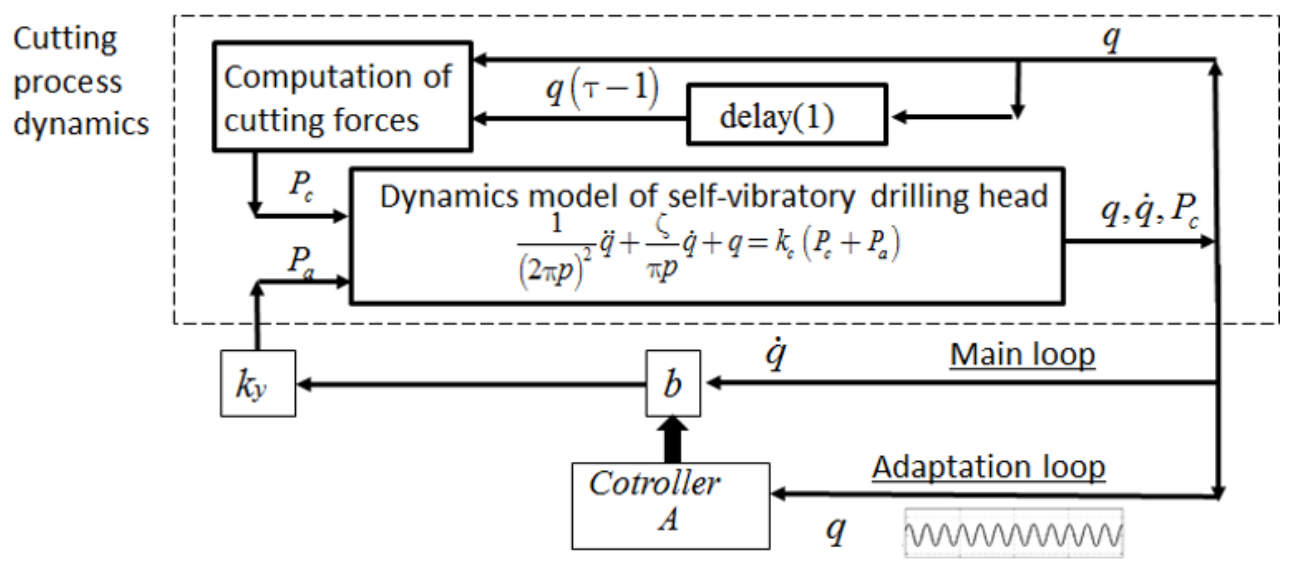

Fig. 3. System flowchart «self-vibratory drilling head-machining process-control system».

The following adaptive algorithm is assigned:

$$
\dot{b}=-c_{1}\left(\frac{A}{A_{0}}-1\right)-c_{2} \frac{\dot{A}}{A_{0}}
$$

where $A$ is current peak-to-peak value, $A_{0}$ is target peak-to-peak value, $c_{1}, c_{2}$ are adaptation parameters. For further calculations assume $A_{0}=1.5, c_{1}=500, c_{2}=2300$.

Simulated results of vibratory drilling process dynamics with and without control are given in Fig. 4, 5. It is apparent that described built-in control provides steady vibratory mode; cutting force $P_{c}$ regularly vanishes, which means the chips are being crushed. 

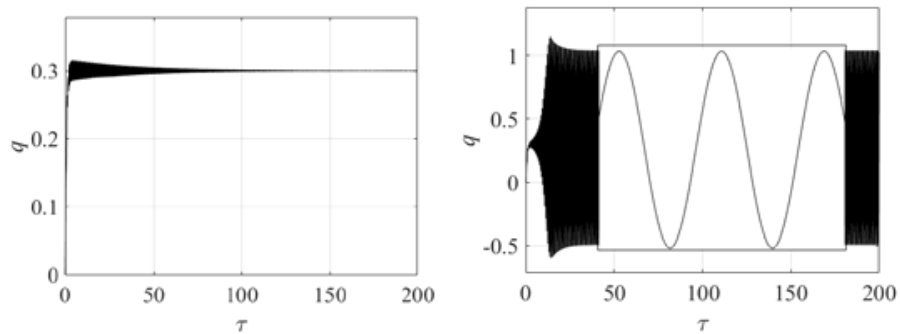

Fig. 4. Time dependence of vibrational displacements of self-vibratory drilling head without (left) and with (right) control.
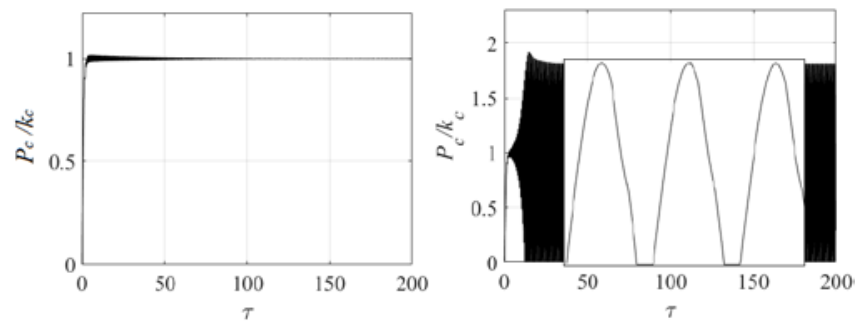

Fig. 5. Time dependence of axial cutting force without (left) and with (right) control.

\section{Study on influence of parameters of signal level resolution of analog-to-digital conversion on controlled vibratory process}

All values used in the adaptive algorithm are digitalized with a signal going through temporal sampling and amplitude quantization. The following part of study estimates the influence of signal amplitude quantization on the controlled process quality. Along with sampling, analog-to-digital conversion (ADC) includes signal limitation in magnitude associated with limited working range of analog-to-digital converter.

Signal quantization could be described by the following nonlinear conversion:

$$
u=\left\{\begin{array}{c}
q_{\min }, q<q_{\min } \\
q_{\min }+\left[\frac{\left(q-q_{\min }\right)}{q_{\max }-q_{\min }} \cdot\left(2^{N}-1\right)\right] \cdot \frac{q_{\max }-q_{\min }}{2^{N}-1}, q_{\max } \geq q \geq q_{\min } \\
q_{\max }, q>q_{\max }
\end{array}\right.
$$

where $u$ is a converted signal; $q_{\min }, q_{\max }$ are, respectively, minimum and maximum values of working range of ADC; $N$ is resolution of ADC. In the calculation, it is assumed that $q_{\min }=-q_{\max }$. Therefore, it is thought that adaptive algorithm input (6) does not receive a peak-to-peak value of $q$, but value $u$ calculated using formula (7).

A joint effect of limitation of removed signal quantity and resolution of ADC is demonstrated in Fig. 6. It is apparent that control aim cannot be achieved when resolution of ADC is low and limitation value $q_{\max }$ is high. Moreover, control aim cannot be achieved when $q_{\max }$ values are too low, meaning that control system does not receive information about amplitude of oscillations, which exceed the low threshold. Single-dimensional dependence relations between defined peak-to-peak value and target value for 8- and 10-bit ADC are given in Fig. 7. 


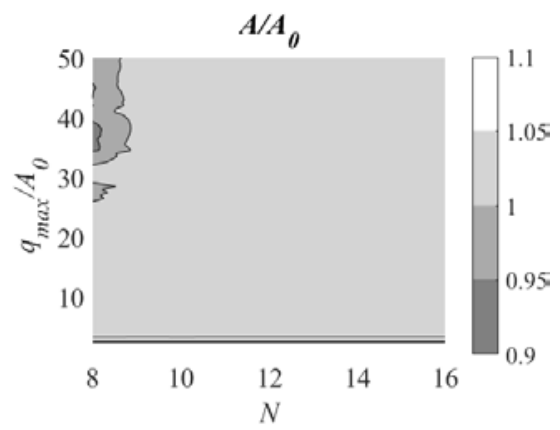

Fig. 6. Dependence relations between peak-to-peak and target value from amplitude quantization parameters.
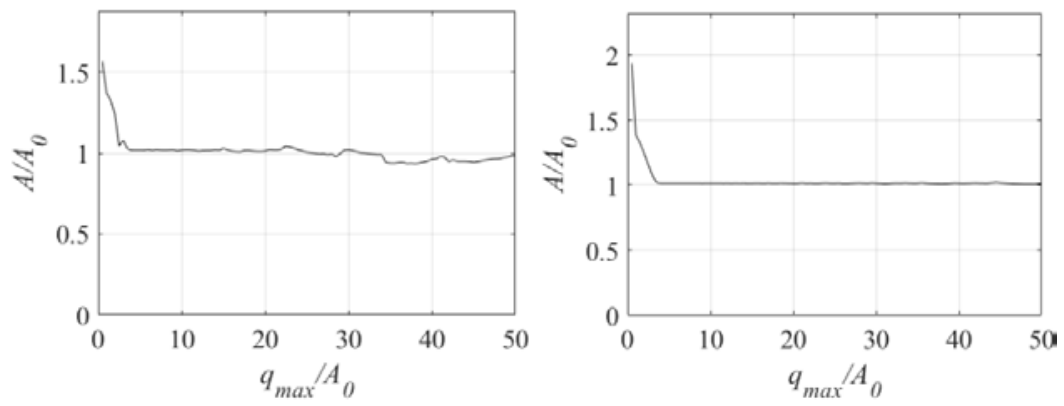

Fig. 7. Dependence relations between peak-to-peak and target value from $q_{\max }$ parameter for 8-bit (left) and 10-bit (right) ADC.

In Fig. 8, three types of axial vibration plots of a tool are compared: ADC is not considered, ADC is considered with low and high limit of sensitivity range. It is seen that character of vibratory process appears unchanged; however, introduction of ADC with low resolution or low response limit causes an essential error in peak-to-peak value.
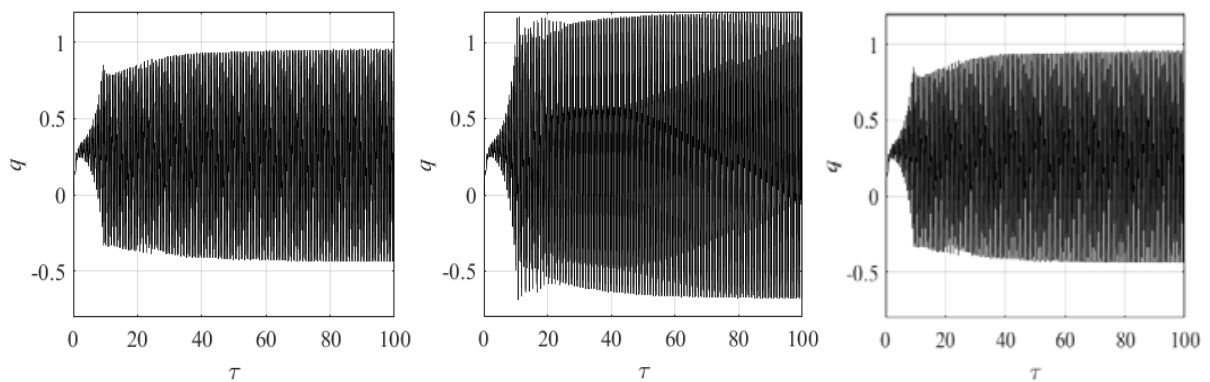

Fig. 8. Comparison of three types of axial vibration plots of a tool: $A D C$ is not considered (left), 8-bit $\mathrm{ADC}$ with $q_{\max } / A_{0}=2$ is introduced (center), 8-bit ADC with $q_{\max } / A_{0}=100$ is introduced (right).

\section{Conclusion}

The current study includes a multi-variant dynamics simulation of a controlled vibratory drilling process considering the influence of analog-to-digital conversion of a measured signal. Mathematic model consists of single-DOF dynamic model of self-vibratory drilling head, phenomenological model of cutting forces, model of surface geometry generation, and control system model with adaptation of feedback gain by peak-to-peak value. It is shown that quality of control cannot be achieved when upper response limit of ADC does 
not exceed tripled target peak-to-peak value. Finally, high value of upper response limit of $\mathrm{ADC}$ may cause degradation of control aim accuracy in 8-bit ADC, however, this effect is not as significant for 10-bit ADC.

The reported study was funded by RFBR according to the research project № 18-31-00147.

\section{References}

1. V. Poduraev, Obrabotka rezaniem s vibratsiyami (Mashinostroenie, Moscow, 1970)

2. A. Gouskov, D. Picard, Sverlil'naya golovka s vibratsionnym effektom (pat. RF 2212984)

3. Y. Altintas, Manufacturing Automation (Cambridge University Press, 2012)

4. S. Voronov, A. Gouskov, I. Ivanov, D. Barysheva, I. Kiselev, Sc. and Ed. Bauman MSTU 12, 842 (2014)

5. I. Kiselev, N. Zhukov, A. Selivanov, D. Barysheva, S. Voronov, A. Gouskov, Proc. Eng. 176, 50 (2017)

6. H. Paris, S. Tichkiewitch, G. Peigne, CIRP Ann. - Man. Tech. 54, 367 (2005)

7. S. Batzer, A. Gouskov, S. Voronov, J. Vibr. Acoust. 123, 435 (2001)

8. A. Gouskov, Razrabotka metodov postroeniya $i$ analiza dinamicheskih modeley tekhnologicheskih protsessov pri mekhanicheskoy obrabotke (Doctoral Diss., 1997)

9. A. Gouskov, S. Voronov, I. Ivanov, S. Nikolaev, D. Barysheva, JVE 17, 3702 (2015)

10. A. Gouskov, S. Voronov, I. Ivanov, S. Nikolaev, D. Barysheva, JVE 17, 4301 (2015) 